\title{
Video Article \\ Generation of 3-D Collagen-based Hydrogels to Analyze Axonal Growth and Behavior During Nervous System Development
}

\author{
Vanessa Gil ${ }^{1,2,3,4}$, José Antonio Del Río 1,2,3,4 \\ ${ }^{1}$ Molecular and Cellular Neurobiotechnology, Institute for Bioengineering of Catalonia (IBEC), The Barcelona Institute of Science and Technology (BIST), Parc Científic \\ de Barcelona \\ ${ }^{2}$ Department of Cell Biology, Physiology, and Immunology, Universitat de Barcelona \\ ${ }^{3}$ Center for Networked Biomedical Research on Neurodegenerative Diseases (CIBERNED) \\ ${ }^{4}$ Institute of Neuroscience, University of Barcelona
}

Correspondence to: Vanessa Gil at vgil@ibecbarcelona.eu, José Antonio Del Río at jadelrio@ibecbarcelona.eu

URL: https://www.jove.com/video/59481

DOI: doi:10.3791/59481

Keywords: Neuroscience, Issue 148, 3-D hydrogel cultures, axonal growth, tissue explants, embryonic nervous system, cell transfection, chemoattraction, chemorepulsion

Date Published: $6 / 25 / 2019$

Citation: Gil, V., Del Río, J.A. Generation of 3-D Collagen-based Hydrogels to Analyze Axonal Growth and Behavior During Nervous System Development. J. Vis. Exp. (148), e59481, doi:10.3791/59481 (2019).

\section{Abstract}

This protocol uses natural type I collagen to generate three-dimensional (3-D) hydrogel for monitoring and analyzing the axonal growth. The protocol is centered on culturing small pieces of embryonic or early postnatal rodent brains inside a 3-D hydrogel formed by the rat tail tendonderived type I collagen with specific porosity. Tissue pieces are cultured inside the hydrogel and confronted to specific brain fragments or genetically-modified cell aggregates to produce and secrete molecules suitable for creating a gradient inside the porous matrix. The steps of this protocol are simple and reproducible but include critical steps to be considered carefully during its development. Moreover, the behavior of growing axons can be monitored and analyzed directly using a phase-contrast microscope or mono/multiphoton fluorescence microscope after fixation by immunocytochemical methods.

\section{Video Link}

The video component of this article can be found at https://www.jove.com/video/59481/

\section{Introduction}

Neuronal axons, ending in axonal growth cones, migrate long distances through the extracellular matrix (ECM) of the embryo over specific pathways to reach their appropriate targets. The growth cone is the distal portion of the axon and it is specialized to sense the physical and molecular environment of the cell ${ }^{1,2}$. From a molecular point of view, growth cones are guided by at least four different molecular mechanisms: contact attraction, chemoattraction, contact repulsion, and chemorepulsion triggered by different axonal guidance cues $\mathrm{s}^{3,4,5,6}$. Contact-mediated processes can be partially monitored in two-dimensional (2D) cultures on micro-patterned substrates (e.g., with stripes ${ }^{7,8}$ or spots ${ }^{9}$ containing the molecules). However, axons can navigate to their target in a non-diffusive manner by sensing several attractive and repulsive molecules from guidepost cells in the environment ${ }^{4,5,10}$. Here, we describe an easy method of 3-D culture to check whether a secreted molecule induces chemorepulsive or chemoattractive effects on developing axons.

The earliest studies aimed to determine the effects of axon guidance cues used explant cultures in three-dimensional (3-D) matrices to generate gradients simulating in vivo conditions ${ }^{11,12}$. This approach, together with in vivo experiments, allowed for the identification of four major families of guidance cues: Netrins, Slits, Semaphorins, and Ephrins ${ }^{4,5,6}$. These molecular cues and other factors ${ }^{13}$ are integrated by the growing axons, triggering the dynamics of adhesion complexes and transducing mechanical forces via the cytoskeleton ${ }^{14,15,16}$. To generate molecular gradients in 3-D cultures for axonal navigation, pioneering researchers used plasma clot substrates ${ }^{17}$, which was also used for organotypic slice preparations $^{18}$. However, in 1958, a new protocol to generate 3-D collagen hydrogels was reported for studying with Maximow's devices ${ }^{19}$, a culture platform, used in several studies suitable for microscopic observations ${ }^{20}$. Another pioneer study reported collagen gel as a tool to embed human fibroblasts for studying the differentiation of fibroblasts into myofibroblasts in wound healing processes ${ }^{21}$. In parallel, Lumsden and Davies applied collagen from the bovine dermis to analyze the putative effect of nerve growth factor (NGF) on the guiding of sensory nerve fibers ${ }^{22}$. With the development of new culture platforms (e.g., multi-well plates) by different companies and laboratories, collagen cultures were adapted to these new devices ${ }^{6,23,24,25,26}$. In parallel, an extract of ECM material derived from the Engelbreth-Holm-Swarm tumor cell line was made commercially available to expand these studies $^{27}$.

Recently, several protocols have been developed to generate molecular gradients with putative roles in axon guidance using 3-D hydrogels (e.g., collagen, fibrin, etc. $)^{28}$. Alternatively, the candidate molecule can be immobilized at different concentration in a porous matrix (e.g., NGF $\left.{ }^{29}\right)$ or generated by culturing in a small region of the 3-D hydrogel cell aggregates secreting the molecule to generate a radial gradient ${ }^{4,23,24,25,26}$. The last possibility will be explained in this protocol. 
The procedure presented here is an easy, fast and highly reproducible method based on the analysis of axonal growth in 3-D hydrogel cultures of the embryonic mouse brain. In comparison with other methods, the protocol is well suited for non-trained researchers and can be fully developed after a short training (1-2 weeks). In this protocol, we first isolate collagen from adult rat tails to further generate 3-D matrices in which genetically-modified cell aggregates are cultured in front of the embryonic neuronal tissue. These cell aggregates form radial chemical gradients of a candidate molecule which elicits a response for the growing axons. Finally, the evaluation of the effects of the molecule on growing axons can easily be performed using a phase contrast microscopy or, alternatively, immunocytochemical methods.

\section{Protocol}

All animal experiments were performed under the guidelines and protocols of the Ethical Committee for Animal Experimentation (CEEA) of the University of Barcelona, and the protocol for the use of rodents in this study was reviewed and approved by the CEEA of the University of Barcelona (CEEA approval \#276/16 and 141/15).

\section{Purification of Rat Tail Collagen}

1. Collect adult Sprague-Dawley rat tails (8-9 weeks old) after sacrificing the animal following ethical guidelines and rinse in $95 \%$ ethanol. Place 2-4 tails on ice $\left(4^{\circ} \mathrm{C}\right)$ and keep them covered with ice during the process.

2. To obtain tail tendons, fix the tail at the most caudal vertebrae of the tail using a hemostat and compress the tail with a second hemostat positioned around 5-7 mm from the first. Break the tail by twisting it sharply with both hemostats. To do this, fold/unfold the vertebrae several times until it breaks.

3. Pull the vertebrae slowly with the hemostat to detach the tendons from their sheath as it comes out. At this moment, cut tendons with small scissors. Keep these tendon pieces in a sterile $100 \mathrm{~mm}$ Petri dish on ice.

4. Repeat the clamping and sliding for the rest of the tail until the tendons are totally extracted.

5. Repeat steps 1.2-1.4 for all the obtained tails

6. Observe the tendons under a microscope. Discard blood vessels by cutting with small scissors and holding with straight fine forceps to improve the tendon's purity and rinse the tendons 3 times with ultrapure water.

7. Collect $3-4 \mathrm{~g}$ of wet tendons. Dissolve the tendons in $150 \mathrm{~mL}$ of $3 \%$ glacial acetic acid at $4{ }^{\circ} \mathrm{C}$ in a $200-250 \mathrm{~mL}$ glass conical flask for $24-36 \mathrm{~h}$, under gentle stirring.

8. Centrifuge at $20,000 \times g$ for $1 \mathrm{~h}$. In parallel, prepare the dialysis tubing membrane by cutting a piece of around $10-15 \mathrm{~cm}$ in length and boil it in ultrapure water containing $1 \mathrm{mM}$ ethylenediaminetetraacetic acid (EDTA) for $15 \mathrm{~min}$. Thereafter, gently rinse the dialysis membrane thoroughly with ultrapure water.

9. After centrifugation, collect the supernatant in the dialysis tubing membrane by decantation. The pellet contains acidic insoluble material (noncollagenous proteins) and the supernatant contains soluble collagen proteins.

10. Dialyze the supernatant against $2 \mathrm{~L}$ of sterile $0.1 \times$ Minimum Essential Medium Eagle (MEM), pH 4.0 in a 2-5 L glass beaker. Dialyze for 3 days. Change $0.1 \times$ MEM solution at least twice a day checking the $\mathrm{pH}$ at every change before using. If $\mathrm{pH}$ has turned basic, modify it with a few drops of $0.1 \mathrm{M}$ acetic acid until $\mathrm{pH}$ is 4.0 .

11. After dialysis, add antibiotics ( $1.5 \mathrm{~mL}$ of penicillin/streptomycin (Pen-Strep)) to the dialyzed collagen solution. Make $5 \mathrm{~mL}$ aliquots of the collagen stock solution and store at $4{ }^{\circ} \mathrm{C}$.

NOTE: From this point, all handling procedures must be performed under sterile conditions in a laminar flow hood.

12. Proceed with the gelation test of the prepared collagen stock solution following the next steps.

1. Since the stock collagen is usually too concentrated, prepare 3 working dilutions $(75 \%, 50 \%$, and $25 \%$ collagen solution) by diluting the stock in $0.1 \times \mathrm{MEM}, \mathrm{pH} 4.0$. The final volume recommended for each working dilution is $5 \mathrm{~mL}$. In each condition, check the protein concentration using a protein colorimetric assay.

2. Place several empty $1.5 \mathrm{~mL}$ conical centrifuge tubes (one for each working dilution), $10 \mathrm{x}$ MEM tubes, $7.5 \%$ sodium bicarbonate solution and different working dilutions of collagen on ice. Wait until these are cooled.

3. Add $40-50 \mu \mathrm{L}$ of $10 \mathrm{x}$ MEM to a cold $\left(4^{\circ} \mathrm{C}\right)$ centrifuge tube. Next, mix it gently with $7-8 \mu \mathrm{L}$ of sodium bicarbonate solution.

4. Add $310-330 \mu \mathrm{L}$ of one of the different collagen dilutions to this tube and mix it gently with the pipette avoiding any bubble formation. Keep the collagen-MEM-sodium bicarbonate mixture on ice $\left(4^{\circ} \mathrm{C}\right)$ for at least 5 min.

5. Pipette $10-25 \mu \mathrm{L}$ of the mixture into a $35 \mathrm{~mm}$ Petri dish.

6. Place the Petri dish in the $\mathrm{CO}_{2}$ incubator set at $37^{\circ} \mathrm{C}$ until the gelation of the hydrogel $( \pm 15-20 \mathrm{~min})$ is observed.

7. Repeat steps 1.12.3-1.12.6 for the remaining collagen dilutions in different Petri dishes.

8. Select the collagen working dilution that renders the best results. NOTE: The best gelled hydrogel should have a uniform translucent texture, grey color and should not be lumpy or stringy. In our experience, a dilution of 3:1 (Collagen: $0.1 \times$ MEM) generates the best experimental results.

\section{Preparation of Cell (COS1) Aggregates Genetically-modified to Secrete a Candidate Molecule in 3-D Collagen Hydrogels}

1. Plate $2 \times 10^{6}$ COS1 cells into a $35 \mathrm{~mm}$ Petri dishes and incubate with complete culture medium composed of $100 \mathrm{~mL}$ of $\mathrm{D}-\mathrm{MEM}$ containing $10 \%(\mathrm{vol} / \mathrm{vol})$ heat-inactivated fetal bovine serum, $0.5 \%(\mathrm{wt} / \mathrm{vol})$ glutamine and $1 \%(\mathrm{wt} / \mathrm{vol})$ Pen/Strep in a cell culture incubator, in order to reach $70-80 \%$ confluency overnight. Prepare one Petri dish for each transfection procedure.

2. The following day transfect COS1 cells with the DNA encoding the candidate molecule (Netrin-1 or Sema3E) using liposome-based transfection method following the manufacturer's instructions.

1. To do this, mix $250 \mu \mathrm{L}$ of serum-free medium and DNA (1-2 $\mu \mathrm{g}$ per condition) to a $1.5 \mathrm{~mL}$ centrifuge tube (DNA tube) and mix. Incubate at room temperature (RT) for $5 \mathrm{~min}$. Prepare a second tube (liposomal tube) by adding $240 \mu \mathrm{L}$ of the serum-free medium and $10 \mu \mathrm{L}$ of the liposomal transfection reagent. Incubate at RT for $5 \mathrm{~min}$. 
2. After incubation, add the content of the DNA-tube to the liposomal tube and mix gently. Now incubate at RT for 15 min. Replace the medium on the cultured cells with $1.5 \mathrm{~mL}$ of the serum-free medium and add the DNA-liposomes mixture to the Petri dish slowly dropwise. Incubate for $3 \mathrm{~h}$ in the $\mathrm{CO}_{2}$ cell culture incubator.

3. After $3 \mathrm{~h}$ of transfection incubation, replace the medium with the complete culture medium and incubate overnight in the incubator.

4. Next day, rinse the cells with $0.1 \mathrm{M}$ Dulbecco's phosphate buffered saline (D-PBS), treat cultures with Trypsin-EDTA (800 $\mu \mathrm{L}$ per each dish for $5-15 \mathrm{~min}$ in the $\mathrm{CO}_{2}$ incubator) and collect detached cells with $15 \mathrm{~mL}$ of complete culture medium.

5. Centrifuge the cells at $4{ }^{\circ} \mathrm{C}$ at $130 \times g$ for $5 \mathrm{~min}$. After centrifugation, remove media and preserve the pellet containing COS1 cells on ice.

6. Repeat steps 1.12.3-1.12.6 to prepare the collagen working mixture.

7. Add $100-150 \mu \mathrm{L}$ of the collagen mixture to the pellet of the transfected cells and mix gently by pipetting up and down and spread $45-50 \mu \mathrm{L}$ of this mixture onto a Petri dish ( $35 \mathrm{~mm}$ diameter) to form a uniform band of collagen-cells of around 1-1.5 $\mathrm{cm}$ in length. Place the dish in the incubator at $37^{\circ} \mathrm{C}\left(5 \% \mathrm{CO}_{2}\right)$ until the gelation $( \pm 15-20 \mathrm{~min})$ is observed.

8. Prepare a second strip containing control cells (mock transfection) in a second culture dish and plate it in the incubator ( $\pm 15-20$ min) and when gelation is completed add 3-4 $\mathrm{mL}$ of warmed $\left(37^{\circ} \mathrm{C}\right) \mathrm{COS} 1$ complete culture medium to each dish containing the gelled collagen-cells strips and keep them in the $\mathrm{CO}_{2}$ cell culture incubator. Thereafter, cut the collagen-cells strips to generate small square pieces (400 to 500 $\mu \mathrm{m}$ in length) using a fine scalpel or a tissue chopper.

9. Transfer all the sections from the same transfection condition to a Petri dish containing 3-3.5 mL of neuronal culture media (NCM) and check under a dissection microscope the quality of the pieces. Again, keep them in the $\mathrm{CO}_{2}$ cell culture incubator.

NOTE: Neuronal culture medium consists of Neurobasal medium containing 1-5\% (vol/vol) heat-inactivated horse serum, $2 \mathrm{mM}$ glutamine, $0.5 \%(\mathrm{wt} / \mathrm{vol})$ glucose, $1 \%(\mathrm{wt} / \mathrm{vol})$ Pen-Strep solution and $0.044 \%(\mathrm{wt} / \mathrm{vol}) \mathrm{NaHCO}_{3}$. Ensure that the $\mathrm{pH}$ is between 7.2-7.3.

\section{Generation of Embryonic Explant for Culture}

1. Sterilize the surgical tools (scissors, scalpel blade handle, straight and curved forceps) by autoclaving following routine sterilization guidelines. In parallel prepare $500 \mathrm{~mL}$ of Hank's balanced salt solution-glucose buffer and 4-5 Petri dishes (100 mm diameter) containing 10 $\mathrm{mL}$ of HBSS-G. Place these plates on ice $\left(4^{\circ} \mathrm{C}\right)$.

2. Sacrifice the pregnant female rat (embryonic day 16.5) outside the sterile area, following the approved ethical procedures. Cut the embryo horns with scissors from the abdominal cavity and place it into a large Petri dish containing cold HBSS-G.

3. Place the dish in the laminar flow hood and extract the embryos with straight forceps. Place them into a new dish containing cold HBSS-G. Next, remove the skin of the embryo using small forceps and carefully dissect the brain using the curved and straight forceps. Place them into a dish containing cold HBSS-G.

4. Under a dissecting microscope, cut the brain in half along the midline to separate both the hemispheres with the scalpel or fine scissors and remove the diencephalon, the meninges and blood vessels from the brain pieces with fine forceps.

5. Repeat steps 3.3-3.4 with the rest of the embryos. Do not delay the dissection for more than $2 \mathrm{~h}$ to preserve the tissue quality.

6. Clean all parts of the tissue chopper with $100 \%$ ethanol (especially the polytetrafluoroethylene (PTFE) cutting plate and the razor blade). Keep the tissue chopper in the laminar flux hood under UV illumination for $15 \mathrm{~min}$.

7. Transfer each tissue piece (e.g., cortex with hippocampus) to the cutting plate of the tissue chopper. For hippocampus, place it perpendicular to the razor blade and obtain tissue sections of 450-500 $\mu \mathrm{m}$ in thickness.

8. Prepare several $35 \mathrm{~mm}$ Petri dishes with 3-4 $\mathrm{mL}$ of complete NCM and transfer tissue pieces from the tissue chopper plate to the Petri dishes. Many dishes may be needed as regions of interest are dissected.

9. Finish the tissue dissection in the complete NCM using fine tungsten needles. Check the quality of the obtained slices under the dissecting microscope. Ensure that the layers are clearly identifiable in the darkfield optics and dissect the region of interest (e.g., CA region of the

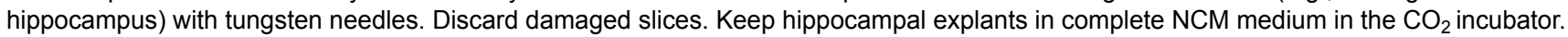

\section{Preparation of 3-D Co-cultures in Collagen Hydrogels}

1. Place several sterile 4-well culture plates in the laminar flow hood and prepare a collagen working mixture as previously indicated in steps 1.12.2-1.12.6.

2. Place $15-20 \mu \mathrm{L}$ of the hydrogel mixture into the bottom of a well to produce a circular collagen base. Repeat this step for the rest of the wells. Do not prepare more than five plates at the same time to avoid excessive liquid evaporation from the hydrogel base.

3. Keep the dishes in the incubator until the complete gelation $( \pm 15-20 \mathrm{~min})$ is observed and take the plates out of the incubator only when the gelation is completed. Check the quality of the gelled collagen.

4. Transfer a small piece of COS1 cell aggregate with a pipette. Place it onto the hydrogel base and place a tissue piece on the same base with a pipette close to the piece of cells aggregate at one explant-size.

5. Prepare a new working collagen mixture on ice as in steps 1.12.2-1.12.6.

6. Gently pipette $15-20 \mu \mathrm{L}$ of this new mixture and cover the explant and cell aggregate. A sandwich-like hydrogel culture will be observed. At this moment, re-orientate the explant with a fine tungsten needle (do not touch the COS1 cell aggregate!), so it faces the cell aggregate at \pm 500-600 $\mu \mathrm{m}$.

7. Return the plate to the incubator until the gelation is observed ( $\pm 10-15 \mathrm{~min})$, and $0.5 \mathrm{~mL}$ of complete NCM supplemented with $2 \%$ B27 supplement and keep cultures for 36 to $48 \mathrm{~h}$ in the incubator $\left(37^{\circ} \mathrm{C}, 5 \% \mathrm{CO}_{2}\right)$.

\section{Fixation of Explant-cell Aggregate Co-cultures and Immunocytochemical Procedure}

1. After $36-48 \mathrm{~h}$ of incubation, remove the medium and rinse with $0.1 \mathrm{M}$ phosphate buffered saline (PBS), $\mathrm{pH} 7.3$. Thereafter, fix the cultures for $1 \mathrm{~h}$ with $4 \%$ paraformaldehyde in $0.1 \mathrm{M}$ phosphate buffer (PB), $\mathrm{pH} 7.3$, at $4{ }^{\circ} \mathrm{C}$.

2. Remove the fixative and gently rinse the cultures $3-4$ times (10-15 min each) in $0.1 \mathrm{M} \mathrm{PB}, \mathrm{pH} 7.3$.

3. Detach the hydrogel sandwich from the bottom of the well with spatula or forceps. Transfer the collagen block with a fine paintbrush to a 6well culture plate containing $0.1 \mathrm{M}$ PBS with $0.5 \%$ non-ionic detergent. 
4. Incubate free-floating hydrogels in the blocking solution ( $10 \%$ serum, $0.5 \%$ non-ionic surfactant, and $0.2 \%$ gelatin in $0.1 \mathrm{M} \mathrm{PBS}$ ) for $2-3 \mathrm{~h}$ at RT with gentle agitation.

5. Rinse 3 times (10-15 min each) with $0.1 \mathrm{M}$ PBS containing $0.5 \%$ non-ionic surfactant.

6. Incubate with primary antibody diluted in PBS containing $5 \%$ serum, $0.5 \%$ non-ionic surfactant, $0.2 \%$ gelatin, and $0.02 \%$ sodium azide. Incubate with the primary antibody for $36-48 \mathrm{~h}$ at $4{ }^{\circ} \mathrm{C}$ on a shaker.

NOTE: Usually an antibody against class III $\beta$-tubulin ( $\alpha$-TUJ-1) (diluted 1:2,000) is used to define axonal growth in 3-D hydrogel cultures.

7. After incubation, rinse as in step 5.5.

8. Incubate with secondary antibody for $4 \mathrm{~h}$ at RT (or $6-7 \mathrm{~h}$ at $4{ }^{\circ} \mathrm{C}$ ) on a shaker diluted in $5 \%$ serum, $0.5 \%$ non-ionic surfactant, and $0.2 \%$ gelatin. A horse anti-mouse biotinylated antibody (diluted 1:200) is used in this experiment.

9. Rinse cultures as in 5.5 .

10. Incubate the cultures for 2 days at $4{ }^{\circ} \mathrm{C}$ with avidin-biotin complex (ABC) solution $1: 100$ diluted in PBS containing $5 \%$ serum, $0.5 \%$ non-ionic surfactant, and $0.2 \%$ gelatin. Alternatively, use horseradish peroxidase (HRP)-tagged streptavidin (diluted 1:300-400) in the same buffer.

11. Rinse cultures as described in 5.5 .

12. Rinse the cultures several times with $0.1 \mathrm{M}$ Tris- $\mathrm{HCl}$ buffer, $\mathrm{pH} 7.6$ for $1 \mathrm{~h}$.

13. Incubate the cultures with $0.03 \%$ of $3,3^{\prime}$-Diaminobenzidine tetrahydrochloride (DAB) solution in $0.1 \mathrm{M} \mathrm{Tris-HCl,} \mathrm{pH} 7.6$.

14. Add 5-8 $\mu \mathrm{L}$ of $1 \% \mathrm{H}_{2} \mathrm{O}_{2}$ and wait 10-15 min. Monitor the development of DAB under a microscope using a 4-10x objective.

15. Stop the reaction by removing $\mathrm{DAB}$ solution with $0.1 \mathrm{M}$ Tris-HCl buffer, $\mathrm{pH}$ 7.6.

16. Rinse the cultures in PBS for 30 min (several changes).

17. Mount the hydrogels onto glass slides using aqueous-based mounting media.

18. Analyze the length and distribution of the axons inside the hydrogel using Sholl analysis plug-in or with NeuriteJ plug-in for ImageJ software ${ }^{30}$.

\section{Representative Results}

Here, we present a widely accessible methodology to study axonal growth in 3-D hydrogel collagen cultures of embryonic mouse nervous system. To this end, we isolated collagen from adult rat tails to generate 3-D matrices in which we cultured genetically-modified cell aggregates expressing Netrin-1 or Sema3E confronted with embryonic neuronal tissue (e.g., CA region of the hippocampus). These cell aggregates formed a radially distributed gradient of the candidate molecule inside the collagen matrix. Finally, to evaluate the neuronal response to different molecules, we labeled the cultures using immunocytochemical methods (e.g., $\alpha$-TUJ-1) and by applying a simple and easy quantification method, we obtained enough data to determine the effect of the putative candidate on axonal behavior.

In our experiment, when hippocampal axons were confronted with Netrin-1, these axons grew preferentially towards the source of Netrin-1 which indicates that Netrin-1 acts as a chemoattractive molecule for these axons (Figure 1B). In contrast, when hippocampal axons where confronted with Sema3E-secreting cells, most of them grew opposite to the cell aggregate indicating that Sema3E is a chemorepulsive molecule for them (Figure 1C). In the control condition (mock transfection), all axons grew radially without any directional preference (Figure 1A). Figure 1D-E are schematic representations of the axonal response and quantification method. After image acquisition, we drew a line in the middle of the explant which delimited the proximal (close to cell aggregate) and the distal (opposite to the cell aggregate) quadrants in order to calculate the proximal/distal ratio (P/D ratio). In control conditions, the axons were equally distributed in both quadrants (radial outgrowth) which indicated a ratio $P / D=1$ (Figure 1D). When explants showed increased number of axons in the proximal quadrant in comparison to the distal (indicating chemoattraction) the ratio was $P / D>1$ (Figure 1E) and when the number of axons was higher in the distal quadrant than in the proximal one (indicating chemorepulsion) the ratio was $P / D<1$ (Figure 1F).

In order to achieve excellent results with this technique, we must make sure that collagen polymerization is homogenous, cell transfection is efficient, and the distance between the tissue explant and the cell aggregate is appropriate (see Discussion).

In conclusion, we can confirm that the generation of 3-D collagen-based hydrogels is a useful technique in order to evaluate axonal growth and behavior responses to candidate guidance molecules which can be playing essential roles in the axonal migration during nervous system development. 


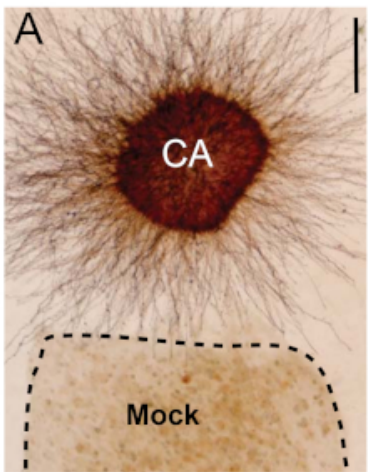

D

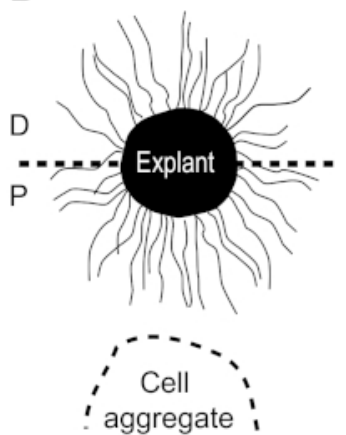

$P / D=1$

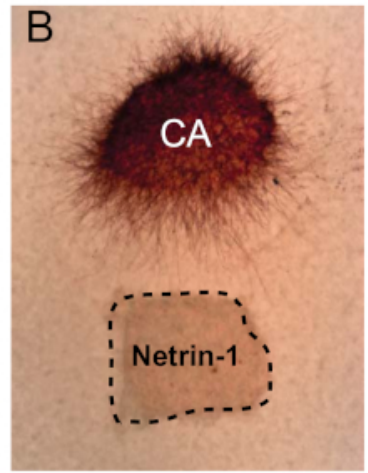

E

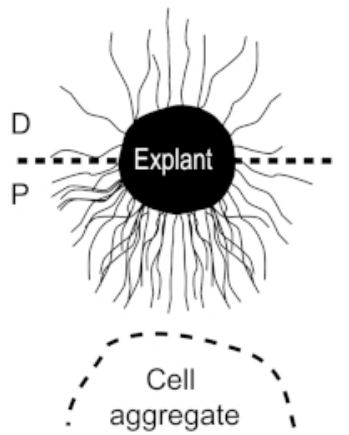

$P / D>1$

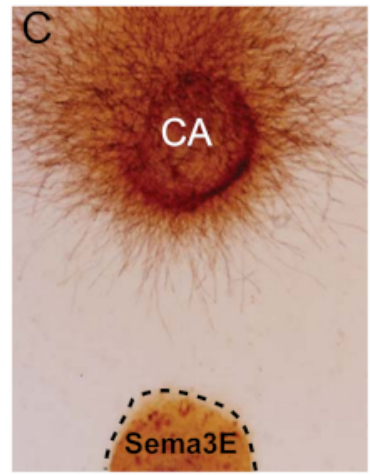

$\mathrm{F}$

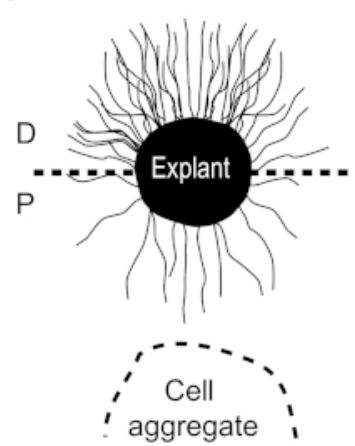

$P / D<1$

Figure 1: Examples of explants growing in 3-D hydrogels in confrontation experiments and quantification methods. (A-C) Explants were obtained from the hippocampal region at E14.5, cultured for $48 \mathrm{~h}$ in vitro, and labeled with $\beta$ III-tubulin $(\alpha-T U J-1)$ by immunostaining. Differences in axonal growth can be observed visually. Please compare (A) with (B-C). (D-E) Schematic representations of the axonal response and quantification method. Dotted line delimits both the proximal $(P)$ and the distal $(D)$ quadrant in order to calculate the ratio P/D. Ratio P/D $=1$ represents a radial pattern of growth (D); $P / D>1$ indicates a chemoattractive response $(E)$, and $P / D<1$ indicates a chemorepulsive effect $(F)$. Abbreviations: $\mathrm{CA}=\mathrm{CA} 1-3$ hippocampal regions; $\mathrm{D}=$ distal quadrant; $\mathrm{P}=$ proximal quadrant. Scale bars $=200 \mu \mathrm{m}(\mathrm{A}-\mathrm{C})$. Please click here to view a larger version of this figure.

\section{Discussion}

The growth of developing axons is mainly invasive and includes ECM degradation and remodeling. Using the procedure presented here, researchers can obtain a homogenous 3-D matrix formed by the natural type I collagen in which axons (or cells) can respond to a chemical gradient secreted by genetically-modified cells as they do in vivo. Different axonal responses to gradients of attractive or inhibitory cues (protein, lipids, etc.) can be easily compared to specific control (mock transfected cells). As advantages, we must mention that tendons are easy to isolate and indeed they can be remnants of animal experimentation. In addition, tendons are highly collagen I concentrated compared to other tissues such as skin or lung ${ }^{31}$.

Although the methodology presented here is simple to perform, there are some steps that need special attention during the process. Concerning collagen extraction, it is imperative to remove unwanted blood vessels and skin debris from tail tendons in order to improve collagen purity and the quality of gelation. Also, it is mandatory to maintain sterile conditions by performing some steps under a sterile laminar flow hood and sterilizing the surgical tools before use. In addition, it is important to maintain the appropriate $\mathrm{pH}$ and temperature conditions of the solutions. For instance, if MEM 10x and bicarbonate solutions are not optimal, the collagen matrix will not polymerize homogeneously, and consequently, the axonal growth and result will be negatively affected. Moreover, if the collagen stock solution is too concentrated or too diluted, the matrices will not gel properly. In our experience, the best collagen stock concentration is approximately $5-5.5 \mathrm{mg} / \mathrm{mL}$ of protein (quantified by a colorimetric protein assay kit) and we use a 3:1 dilution (Collagen: $0.1 \times$ MEM) to obtain perfect hydrogel matrices. Regarding cell transfection and cell aggregate formation, it is important to maintain sterile conditions and avoid possible contamination, for example, purifying plasmid vectors with endotoxin-free plasmid DNA purification kits is mandatory. Also, we must emphasize that the transfection conditions vary depending on the cell type, passage number, and the plasmid characteristics. Here, we have reported the optimal and routine conditions in our hands. Therefore, researchers should test the recommended concentrations indicated by the manufacturer or adjust them to determine their own optimal conditions.

Regarding the problems that may arise with this technique, we must consider that sometimes the 3-D matrices do not present the expected homogeneous gel-like structure. In this case, it is important to check the temperature and pH condition of the solutions and discard them in case it is incorrect. Also, it is recommended to perform a quality control test such as denaturing polyacrylamide gel electrophoresis (SDS-PAGE) under reducing conditions to validate the purity of collagen stock preparation. With this approach, pure and undamaged type I collagen shows a 
typical migration pattern consisting of 2 monomeric $\alpha$ chains ( $\alpha 1$ and $\alpha 2), 3$ dimeric $\beta$ chains $(\beta 11, \beta 12$, variant $\beta 11)$, and 1 trimeric $y$ chain ${ }^{32,33}$. If the obtained collagen does not fit this pattern, it should not be used. Lastly, after immunostaining, axons can appear radially distributed when confronted with cells secreting a chemorepulsive or chemoattractive molecule. In this situation, the efficiency of transfection must be checked by performing a dot blotting technique on the proper co-culture system (if the DNA plasmids are alkaline phosphatase-tagged) or by processing the culture media after transfection by western blotting. A limitation to consider is that the distance between the cell aggregate and tissue explant is crucial. If they are very far apart, we will not be able to see any clear effect of the secreted molecule on the tissue explant, but if they are very close to each other, the effect will be too strong to be considered as a good result. From our experience, the appropriate distance is around one explant-size $(400-500 \mu \mathrm{m})$ because the molecular gradient generated by the cell aggregate will extend radially from along $400-500 \mu \mathrm{m}$ after $24 \mathrm{~h}$ in culture.

Alternatively, one can use commercial tumor-derived ECM extract instead of rat tail collagen. In that case, all the procedures must be performed at between 4 and $10^{\circ} \mathrm{C}$, since the gelation of commercial ECM extract is temperature-dependent. Thus, special care should be taken to ensure all culture dishes, pipette tips, culture media, and solutions are maintained at $4{ }^{\circ} \mathrm{C}$.

Finally, although the method presented here is mainly associated with the analysis of neuronal functions such as axonal growth or neuronal migration, it also becomes a useful technique for the pharmacological screening, adhesion assays, in vitro fibrillation experiments and tissue engineering strategies ${ }^{34,35,36}$.

\section{Disclosures}

The authors have nothing to disclose.

\section{Acknowledgments}

The authors thank Tom Yohannan for the editorial advice and M. Segura-Feliu for the technical assistance. This work was funded by the CERCA Programme and by the Commission for Universities and Research of the Department of Innovation, Universities, and Enterprise of the Generalitat de Catalunya (SGR2017-648). This work was funded by the Spanish Ministry of Research, Innovation and University (MEICO) through BFU2015-67777-R and and RTI2018-099773-B-100, the Spanish Prion Network (Prionet Spain AGL2017-90665-REDT), and the Institute Carlos III, CIBERNED (PRY-2018-2).

\section{References}

1. Ramón y Cajal, S. Les nouvelles idées sur la structure du système nerveux chez l'homme et chez les vertébrés. (1894).

2. Ramón y Cajal, S. Nuevo concepto de la histología de los centros nerviosos. (1893).

3. Marin, O., Valiente, M., Ge, X., Tsai, L. H. Guiding neuronal cell migrations. Cold Spring Harbor Perspectives in Biology. 2 (2), a001834 (2010).

4. Tessier-Lavigne, M., Goodman, C. S. The molecular biology of axon guidance. Science. 274 (5290), 1123-1133 (1996)

5. Dickson, B. J. Molecular mechanisms of axon guidance. Science. 298 (5600), 1959-1964 (2002).

6. Kolodkin, A. L., Tessier-Lavigne, M. Mechanisms and molecules of neuronal wiring: a primer. Cold Spring Harbor Perspectives in Biology. 3 (6), a001727 (2011)

7. Rosentreter, S. M. et al. Response of retinal ganglion cell axons to striped linear gradients of repellent guidance molecules. Journal of Neurobiology. 37 (4), 541-562 (1998).

8. Knoll, B., Weinl, C., Nordheim, A., Bonhoeffer, F. Stripe assay to examine axonal guidance and cell migration. Nature Protocols. 2 (5), 1216-1224 (2007).

9. von Philipsborn, A. C. et al. Growth cone navigation in substrate-bound ephrin gradients. Development. 133 (13), $2487-2495$ (2006).

10. Chen, H., He, Z., Tessier-Lavigne, M. Axon guidance mechanisms: semaphorins as simultaneous repellents and anti-repellents. Nature Neuroscience. 1 (6), 436-439 (1998).

11. Jessell, T. M., Sanes, J. R. Development. The decade of the developing brain. Current Opinion in Neurobiology. 10 (5), $599-611$ (2000).

12. Serafini, T. et al. The netrins define a family of axon outgrowth-promoting proteins homologous to C. elegans UNC-6. Cell. 78 (3), $409-424$ (1994).

13. Charron, F., Tessier-Lavigne, M. Novel brain wiring functions for classical morphogens: a role as graded positional cues in axon guidance. Development. 132 (10), 2251-2262 (2005).

14. 1Fournier, M. F., Sauser, R., Ambrosi, D., Meister, J. J., Verkhovsky, A. B. Force transmission in migrating cells. Journal of Cell Biology. 188 (2), 287-297 (2010).

15. Dent, E. W., Gertler, F. B. Cytoskeletal dynamics and transport in growth cone motility and axon guidance. Neuron. 40 (2), $209-227$ (2003).

16. Lowery, L. A., Van Vactor, D. The trip of the tip: understanding the growth cone machinery. Nature Reviews Molecular Cell Biology. 10 (5), 332-343 (2009).

17. Castellani, V. B., in Protocols for Neuronal Cell Culture. Humana Press Inc, (2001).

18. Gahwiler, B. H. Organotypic monolayer cultures of nervous tissue. Journal of Neuroscience Methods. 4 (4), $329-342$ (1981).

19. Bornstein, M. B. Reconstituted rattail collagen used as substrate for tissue cultures on coverslips in Maximow slides and roller tubes. Laboratory Investigation. 7 (2), 134-137 (1958).

20. Billings-Gagliardi, S., Wolf, M. K. A simple method for examining organotypic CNS cultures with Nomarski optics. In Vitro. 13 (6), $371-377$ (1977).

21. Bell, E., Ivarsson, B., Merrill, C. Production of a tissue-like structure by contraction of collagen lattices by human fibroblasts of different proliferative potential in vitro. Proceedings of the National Academy of Science U. S. A. 76 (3), 1274-1278 (1979).

22. Lumsden, A. G., Davies, A. M. Earliest sensory nerve fibres are guided to peripheral targets by attractants other than nerve growth factor. Nature. 306 (5945), 786-788 (1983). 
23. Chedotal, A. et al. Semaphorins III and IV repel hippocampal axons via two distinct receptors. Development. 125 (21), $4313-4323$ (1998).

24. Kennedy, T. E., Serafini, T., de la Torre, J. R., Tessier-Lavigne, M. Netrins are diffusible chemotropic factors for commissural axons in the embryonic spinal cord. Cell. 78 (3), 425-435 (1994).

25. Klein, R. Eph/ephrin signaling in morphogenesis, neural development and plasticity. Current Opinion in Cell Biology. 16 (5), $580-589$ (2004).

26. Wang, K. H. et al. Biochemical purification of a mammalian slit protein as a positive regulator of sensory axon elongation and branching. Cell. 96 (6), 771-784 (1999).

27. Emonard, H., Grimaud, J. A., Nusgens, B., Lapiere, C. M., Foidart, J. M. Reconstituted basement-membrane matrix modulates fibroblast activities in vitro. Journal of Cell Physiology. 133 (1), 95-102 (1987).

28. Knapp, D. M., Helou, E. F., Tranquillo, R. T. A fibrin or collagen gel assay for tissue cell chemotaxis: assessment of fibroblast chemotaxis to GRGDSP. Experimental Cell Research. 247 (2), 543-553 (1999).

29. Kapur, T. A., Shoichet, M. S. Immobilized concentration gradients of nerve growth factor guide neurite outgrowth. Journal of Biomedical Materials Research Part A. 68 (2), 235-243 (2004).

30. Torres-Espin, A., Santos, D., Gonzalez-Perez, F., del Valle, J., Navarro, X. Neurite-J: an image-J plug-in for axonal growth analysis in organotypic cultures. Journal of Neuroscience Methods. 236 26-39 (2014).

31. Balestrini, J. L. et al. Comparative biology of decellularized lung matrix: Implications of species mismatch in regenerative medicine. Biomaterials. 102 220-230 (2016).

32. Qian, J. et al. Kinetic Analysis of the Digestion of Bovine Type I Collagen Telopeptides with Porcine Pepsin. Journal of Food Science. 81 (1), C27-34 (2016).

33. Eyre, D. R., Weis, M., Hudson, D. M., Wu, J. J., Kim, L. A novel 3-hydroxyproline (3Hyp)-rich motif marks the triple-helical C terminus of tendon type I collagen. Journal of Biological Chemistry. 286 (10), 7732-7736 (2011).

34. Garnotel, R. et al. Human blood monocytes interact with type I collagen through alpha $x$ beta 2 integrin (CD11c-CD18, gp150-95). Journal of Immunology. 164 (11), 5928-5934 (2000).

35. Montolio, M. et al. A semaphorin 3A inhibitor blocks axonal chemorepulsion and enhances axon regeneration. Chemistry and Biology. 16 (7), 691-701 (2009).

36. Garcia-Gareta, E. Collagen gels and the 'Bornstein legacy': from a substrate for tissue culture to cell culture systems and biomaterials for tissue regeneration. Experimental Dermatology. 23 (7), 473-474 (2014). 